\title{
Identification of Hammerstein-Wiener Systems Including Backlash Input Nonlinearities
}

\author{
F. Giri ${ }^{1}$, A. Brouri ${ }^{2}$, F. Ikhouane ${ }^{3}$, F.Z. Chaoui ${ }^{4}$, A. Radouane ${ }^{4}$ \\ ${ }^{1}$ Normandie Université, UNICAEN, GREYC UMR CNRS, 14032 Caen, France \\ ${ }^{2}$ Université Moulay Ismail, ENSAM, L2MC, Meknes, Morocco \\ ${ }^{3}$ Universitat Politecnica de Catalunya, Barcelona, Spain \\ ${ }^{4}$ Université Mohamed V, ENSET, Rabat, Marocco
}

\begin{abstract}
Standard Hammerstein-Wiener models consist of a linear subsystem sandwiched by two memoryless nonlinearities. Presently, the input nonlinearity is allowed to be a memory operator of backlash type and both input and output nonlinearities are polynomial and may be noninvertible. The linear subsystem may be parametric or not, continuous- or discrete-time. A two-stage identification method is developed such the parameters of all nonlinear elements are estimated first using the KozenLandau polynomial decomposition algorithm. The obtained estimates are then based upon in the identification of the linear subsystem, making use of suitable pre- ad post-compensators.

Keywords: nonlinear system identification, Hammerstein-Wiener systems, backlash operator, polynomial decomposition.
\end{abstract}

\section{INTRODUCTION}

Hammerstein-Wiener models (Fig. 1) have proved to be suitable in modelling a wider class of physical systems including chemical processes, ionospheric dynamics (Palanthandalam-Madapusi et al., 2005), submarine detection systems (Abrahamsson et al., 2007), RF power amplifiers (Taringou et al., 2010). Compared to the simpler Hammerstein and Wiener models (Giri and Bai, 2010), the problem of Hammerstein-Wiener system identification is much more complex and relatively few works have focused on. Most proposed identification methods mach two main different approaches. The first one is commonly referred to over-parameterization approach (e.g. Bai, 2010; Schoukens et al., 2012). It consists in defining first an over-sized vector involving lumped parameters. This vector is estimated using variants of the least-squares algorithm while the true system parameters are recovered from the lumped parameters using SVD like procedures. The output nonlinearity is required to be invertible and its inverse must have nonzero leading coefficients. Its optimality is widely discussed in (Bai, 2010). The second approach is referred to iterative optimization (Ni et al., 2012; Vörös, 2004; Schoukens et al., 2012). The main underlying idea is to get benefit of the fact that the unknown parameters come bi-linearly in the system overparameterization mentioned in the previous approach. Then, the linear subsystem parameters, on one hand, and the parameters of the nonlinear elements, on the other hand, are estimated in several iterations according to relaxation principle. Specifically, initial estimates of the nonlinear parameters are supposed to be available and substituted to the true parameters in the system over-parameterization. Then, the identification problem boils down to the estimation of the linear subsystem parameters which can be dealt with using variants of the least-squares or the instrumental variable algorithms, depending on the linear subsystem structure. In turn, the estimates thus obtained are substituted to their true values in the system over-parameterization making possible the estimation of the nonlinear parameters using nonlinear least-squares estimators. The above process is repeated a number of times until some criteria is satisfied. This approach requires the output nonlinearity to be invertible and generally leads to suboptimal solutions. In addition to the previous main approaches, available identification methods for Hammerstein-Wiener include blind methods (e.g. Bai, 2002), frequency methods (e.g. Crama and Schoukens, 2004), and stochastic methods (e.g. Wang and Ding, 2008).

In this paper, the identification problem is addressed in presence of nonlinear elements. Specifically, the input nonlinearity is allowed to be a memory operator of backlash type and the output nonlinearity is not necessarily invertible. The borders of the backlash operator and the output nonlinearity are polynomials of known degrees but arbitraryshape. Interestingly, the linear subsystem may be parametric or not, continuous- or discrete-time. A new two-stage identification method is designed that determines first all unknown parameters of the system nonlinear elements. This stage relies upon the polynomial decomposition algorithm of Kozen and Landau (1989). The obtained parameter estimates are used in the second stage to identify the linear subsystem. This is carried out getting benefit of the fact that the input and output nonlinearities are locally invertible, due to their polynomial nature. Local inverse operators are constructed and used as pre- and post-compensators so that the resulting augmented system boils down, within adequate experiments, to the linear part of the initial Hammerstein-Wiener system. Then, the identification of this linear subsystem can be coped with using available methods. 
The paper is organized as follows: relevant mathematical tools are described in Section 2; the identification problem is formulated in Section 3; Section 4 is devoted to the identification of the system nonlinear elements and the linear subsystem identification is discussed in Section 5.

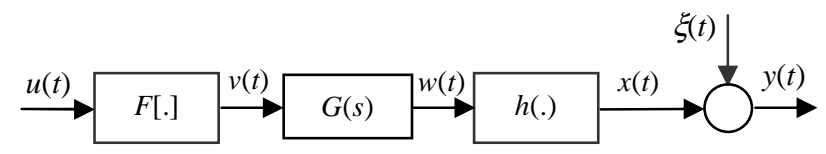

Fig. 1. Hammerstein-Wiener Model structure with a backlash input nonlinearity

\section{MATHEMATICAL PRELIMINARIES}

\subsection{Polynomial Decomposition}

Given a couple of functions, $f: D_{f} \subset \mathbf{R} \rightarrow \mathbf{R}$ and $h: D_{h} \subset \mathbf{R} \rightarrow \mathbf{R}$, one defines the composed function $h \circ f: D_{h \circ f} \subset \mathbf{R} \rightarrow \mathbf{R}$ such that, $h \circ f(x)=h(f(x))$ and $D_{h \circ f}=D_{h} \cap f\left(D_{f}\right)$. Now, consider any monic polynomial,

$$
p(x)=x^{n_{p}}+a_{n_{p}-1} x^{n_{p}-1}+\ldots+a_{1} x+a_{0}, \quad\left(n_{p} \geq 2\right)
$$

with known real coefficients. Suppose there exists a decomposition $p=h \mathrm{o} f$ where:

$$
\begin{aligned}
& f(x)=x^{n_{f}}+b_{n_{f}-1} x^{n_{f}-1}+\ldots+b_{1} x+b_{0}, \quad\left(n_{f} \geq 1\right) \\
& h(x)=x^{n_{h}}+c_{n_{h}-1} x^{n_{h}-1}+\ldots+c_{1} x+c_{0}, \quad\left(n_{h} \geq 1\right)
\end{aligned}
$$

with $n_{p}=n_{f} \times n_{h}$. The degrees $\left(n_{f}, n_{h}\right)$ are known integers but the real coefficients (the $b_{i}$ 's and the $c_{i}$ 's) are not. We are interested in finding these coefficients. The following algorithm, by Kozen and Landau (1989), is one of the most powerful.

Table 1. Polynomial Decomposition Algorithm

Given a polynomial $p$ of the form (1a), its components $(f, h)$ of the form (1b-c) are found following the next steps.

Step 1: Set: $q_{0}=x^{n_{f}}$ and $b_{n_{f}}=1$. Let $l_{1}$ denotes the coefficient of $x^{n_{f} n_{h}-1}$ in $\left(q_{0}\right)^{n_{h}}=x^{n_{f} n_{h}}$ (i.e. $\left.l_{1}=0\right)$. Then, set: $b_{n_{f}-1}=\frac{a_{n_{p}-1}-l_{1}}{n_{h}}=\frac{a_{n_{p}-1}}{n_{h}}$ and $k=2$.

Step 2: Set: $q_{k-1}=q_{k-2}+b_{n_{f}-k+1} x^{n_{f-k+1}}$ and do:

* Compute $\left(q_{k-1}\right)^{n_{h}}$ and determine $l_{k}$ denoting the coefficient of $x^{n_{f} n_{h}-k}$ in $\left(q_{k-1}\right)^{n_{h}}$.

* Calculate: $b_{n_{f-k}}=\frac{a_{n_{p}-k}-l_{k}}{n_{h}}$.

Step 3: for $k=3$ to $n_{f}$ repeat step 2 .

Step 4: Set the triangular matrix A, where $A(i+1, j+1)$ $\left(0 \leq i, j \leq n_{h}\right)$ is the coefficient of $x^{i n_{f}}$ in $\left(q_{n_{f}}=f(x)\right)^{j}$.
Knowing that: $n_{p}=n_{f} \times n_{h}$ and $a_{n_{p}}=c_{n_{h}}=1$, solve the system: $A\left(c_{0} \ldots c_{n_{h}-1} c_{n_{h}}\right)^{T}=\left(\begin{array}{lll}a_{0} & a_{n_{f}} & a_{2 n_{f}} \ldots a_{n_{f} \times n_{h}}\end{array}\right)^{T}$.

Proposition 1 (Kozen and Landau, 1989).

1) The polynomial decomposition of Table 1 is an $O\left(n_{p}^{2} n_{h}\right)$ complexity algorithm determining a couple of components $(f, h)$ satisfying $p=h \circ f$ provided this decomposition is possible.

2) When the decomposition is possible, the couple of components $(f, h)$ is not unique. Then, the set of solutions is defined by $\bar{f}(x)=\gamma f(x)+\gamma \beta$ and $\bar{h}(x)=h\left(\frac{x}{\gamma}-\beta\right)$, where the scalars $\beta$ and $\gamma \neq 0$ are arbitrary

Remark 1. Note that the matrix $A$ in Step 4 of Table 1 is invertible because it is triangular with all diagonal elements being equal to 1

\subsection{Backlash and Backlash-Inverse Operators}

A backlash operator is a memory element characterized by a couple of functions $\left(f_{a}, f_{d}\right)$, called its borders, and is denoted $B\left(f_{a}, f_{d}\right)$. When submitted to an input signal $u(t)$, it generates an output signal $v(t)$ defined as follows:

$v(t)=\left\{\begin{aligned} f_{a}(u(t)) & \text { if } \dot{u}(t)>0 \text { and } v\left(t^{-}\right)=f_{a}\left(u\left(t^{-}\right)\right) \\ f_{d}(u(t)) & \text { if } \dot{u}(t)<0 \text { and } v\left(t^{-}\right)=f_{d}\left(u\left(t^{-}\right)\right) \\ v\left(t^{-}\right) & \text {otherwise }\end{aligned}\right.$

The couple $\left(f_{a}, f_{d}\right)$ must satisfy the operational condition:

$\forall x, \exists z<x: f_{d}(z)=f_{a}(x)$

$$
\text { and } \forall x, \exists z>x: f_{a}(z)=f_{d}(x)
$$

In the sequel, condition (4) is supposed to be fulfilled whenever a backlash operator $B\left(f_{a}, f_{d}\right)$ is involved.

A backlash-inverse operator is also a memory element characterized by a couple of functions $\left(f_{a}, f_{d}\right)$, called its borders, and is denoted $B I\left(f_{a}, f_{d}\right)$. When submitted to an input signal $u(t)$, it generates an output signal $v(t)$ defined as follows:

$$
v(t)= \begin{cases}f_{a}(u(t)) & \text { if } \dot{u}(t)>0 \\ f_{d}(u(t)) & \text { if } \dot{u}(t)<0\end{cases}
$$

This definition entails no condition on the couple $\left(f_{a}, f_{d}\right)$ except for the obvious property:

$\left|f_{d}(x)\right|<\infty \Leftrightarrow\left|f_{a}(x)\right|<\infty$, for all $x$.

Proposition 2 (Compositions involving memory operators). Consider an operator $B\left(f_{a}, f_{d}\right)$, with $\left(f_{a}, f_{d}\right)$ any pair of functions satisfying (4). Then, one has:

$\left.1^{\circ}\right)$ For any function $h: \mathbf{R} \rightarrow \mathbf{R}:$

$$
h \circ B\left(f_{a}, f_{d}\right)=B\left(h_{a} \circ f_{a}, h_{d} \circ f_{d}\right)
$$


2) Suppose $f_{a}, f_{d}$ are similarly monotonic on some domain $D_{f} \subset \mathbf{R}$. then, one has on $f_{a}^{-1}\left(D_{f}\right) \cap f_{d}^{-1}\left(D_{f}\right)$ :

$$
\begin{aligned}
& X\left(f_{a}, f_{d}\right) \circ B I\left(f_{a}^{-1}, f_{d}^{-1}\right)=I, \text { if } f_{a}, f_{d} \text { are increasing } \\
& X\left(f_{a}, f_{d}\right) \circ B I\left(f_{d}^{-1}, f_{a}^{-1}\right)=I, \text { if } f_{a}, f_{d} \text { are decreasing }
\end{aligned}
$$
for $X \in\{B, B I\}$ with $I$ being the identity operator. That is, for any signal $u(t)$ such that $u(t) \in f_{a}^{-1}\left(D_{f}\right) \cap f_{d}^{-1}\left(D_{f}\right)$, one has:

$$
\left(B\left(f_{a}, f_{d}\right) \circ B I\left(f_{a}^{-1}, f_{d}^{-1}\right)\right)[u](t)=u(t), \text { for all } t \geq 0
$$

\section{IDENTIFICATION PROBLEM STATEMENT}

Standard Hammerstein-Wiener systems consists of a linear subsystem, with transfer function $G(s)$, sandwiched by two memoryless nonlinearities (Fig. 1). In this study, the input nonlinearity $F[$.$] is allowed to be a memory operator of$ backlash type i.e. $F=B\left(f_{a}, f_{d}\right)$ for some couple of functions $\left(f_{a}, f_{d}\right)$. The output nonlinearity $h($.$) is$ memoryless and so is entirely characterized by a single function, denoted $h($.$) . Presently, all functions are$ polynomials of the form:

$$
\begin{aligned}
& f_{a}(x)=a_{n} x^{n}+\ldots+a_{1} x+a_{0} \\
& f_{d}(x)=d_{n} x^{n}+\ldots+d_{1} x+d_{0} \\
& h(x)=b_{m} x^{m}+\ldots+b_{1} x+b_{0}
\end{aligned}
$$

Analytically, the system is described by the following equations:

$$
\begin{aligned}
& v=F[u], W(r)=G(r) V(r) \\
& W(r)=G(r) V(r) \text { with } r=s \text { or } z \\
& x=h(w), \quad y=x+\xi
\end{aligned}
$$

where $V(r), W(r)$ denote either the Laplace transforms (continous-time case) or the $z$-transforms (discrete-time case) of the inner signals $v(t), w(t) ; G(r)$ is the transfer function of the linear subsystem. The only measurable signals are the system input $u(t)$ and output $y(t)$. The equation error $\xi(t)$ accounts for external disturbances or measurement noise. Because of space limitation, the rest of the expose is conducted in the simpler case $\xi(t)=0$.

The following assumptions complete the system description: A1. The structure of $G(s)$ is not necessarily known but this is BIBO stable and its static gain is nonzero i.e. $G(0) \neq 0$.

A2. The borders $\left(f_{a}, f_{d}\right)$ are of known degree $n$ and satisfy the backlash operational condition (4). This particularly implies that $\operatorname{sgn}\left(a_{n}\right)=\operatorname{sgn}\left(d_{n}\right)$.

A3. The output nonlinearity $h($.$) is an arbitrary polynomial$ but its degree $m$ is known.

Except for the above assumptions, the functions $G(r)$, $\left(f_{a}, f_{d}\right)$ and $h$ are arbitrary. In particular, the transfer function $G(s)$ may by infinite order. The functions $\left(f_{a}, f_{d}\right)$ are arbitrary-shape as long as condition (4) is fulfilled.
Therefore, they can be noninvertible and crossing. The last feature makes possible to handle, using the same identification method, also the memoryless case $f_{a}=f_{d}$. Interestingly, it is not required to know a priori the exact type of the nonlinear operator $F[$.$] , being backlash or backlash-$ inverse. In turn the function $h$ is allowed to be noninvertible.

We aim at designing an identification method that is able to provide accurate estimates of: (i) the nonlinear operator parameters $\left(a_{i}, d_{i} ; i=1 \ldots n\right)$ and $\left(b_{i} ; i=1 \ldots m\right)$; (ii) and the linear subsystem transfer function.

The identification problem thus defined does not have a unique solution. Indeed, one can easily check that, in addition to the triplet $(F[],. G(r), h()$.$) used in the system description$ (7a-b), any triplet $(\bar{F}[],. \bar{G}(r), \bar{h}()$.$) defined as follows is also$ model:

$$
\begin{aligned}
& \bar{F}[x]=k_{1} F[x]-k_{1} k_{0} \\
& \bar{G}(r)=\frac{G(r)}{k_{1} k_{2}} \\
& \bar{h}(x)=h\left(k_{2}\left(x+k_{0}\right)\right)
\end{aligned}
$$

whatever the real triplet $\left(k_{0}, k_{1}, k_{2}\right)$ with $\left(k_{1}, k_{2}\right)$ nonzero. It is readily checked that the operators $\bar{F}$ [.] and $\bar{h}($.) are of the same nature as $F[$.$] and h($.$) . That is, the former is a$ backlash type, bordered by two polynomials, and the second is a memoryless polynomial function. Let the borders of $\bar{F}[$.] be denoted $\left(\bar{f}_{a}, \bar{f}_{d}\right)$. It is readily checked that:

$$
\begin{aligned}
& \bar{f}_{a}(x)=k_{1} f_{a}(x)-k_{1} k_{0}, \bar{f}_{d}(x)=k_{1} f_{d}(x)-k_{1} k_{0} \\
& \bar{h}(x)=h\left(k_{2}\left(x+k_{0}\right)\right)
\end{aligned}
$$

We get benefit from this model plurality by making the system identification problem a bit simpler. To reach this goal, it will prove judicious to focus on the model $(\bar{F}[],. \bar{G}(s), \bar{h}()$.$) characterized by the following property:$

$$
\begin{aligned}
& a_{0} k_{1}-k_{1} k_{0}=0 \text { and } a_{n} k_{1}=1 \\
& \bar{G}(0)=\frac{G(0)}{k_{1} k_{2}}=1 \quad \text { if } \quad r=s \\
& \bar{G}(1)=\frac{G(1)}{k_{1} k_{2}}=1 \quad \text { if } r=z
\end{aligned}
$$

Property (15a) means that $\bar{f}_{a}$ is made monic and its lower degree coefficient is zero. Property (11b) means that $\bar{G}(r)$ is made unit static gain. Clearly, the two equations (11a-b) determine uniquely the scalars $\left(k_{0}, k_{1}, k_{2}\right)$ which entails the uniqueness of the model $(\bar{F}[],. \bar{G}(r), \bar{h}()$.$) . To avoid$ multiplying notations, the unique model satisfying (11a-b) will sill be denoted $(F[],. G(r), h()$.$) . It turns out using (11a-$ b) and $(7 \mathrm{a}-\mathrm{c})$, that the system model $(8 \mathrm{a}-\mathrm{b})$ is characterized by a triplet $(F[],. G(r), h()$.$) satisfying the following$ properties:

$G(0)=1$ if $r=s \quad$ or $\quad G(1)=1$ if $r=z$ 
$a_{n}=1$ and $a_{0}=0$

\section{IDENTIFICATION OF THE NONLINEAR ELEMENTS}

\subsection{Data acquisition experiments}

The above observations are made beneficial in the design of an experimental protocol to identify the two functions $h o f_{a}$ and $h \circ f_{d}$. This protocol involve two main stages, referred to ascendant and descendant. Each stage involves a series of constant inputs.

Ascendant experimental stage. The system is successively excited by $N$ constant inputs with amplitudes $U_{1}<U_{2}<\ldots<U_{N}$, where:

$N>n+m$

Practically, one first applies the input $u(t)=U_{1}$ over a sufficiently large time interval so that the transient dynamic effect of $G(r)$ vanishes, leading to constant asymptotic values of all system signals i.e.

$u(t)=U_{1} \Rightarrow v(t) \approx w(t) \approx W_{1}, \quad x(t) \approx X_{1}=h\left(W_{1}\right)$

for some scalar $W_{1}$. Similar experiments are repeated yielding, for $i=2 \ldots N$ :

$u(t)=U_{i} \Rightarrow v(t) \approx w(t) \approx W_{i}, \quad x(t) \approx X_{i}=h\left(W_{i}\right)$

Now, since $F[$.] is a backlash operator and the amplitude sequence $U_{i}(i=1 \ldots N)$ is increasing, there is an integer $1 \leq i_{a} \leq N$ such that:

$W_{i}=W_{1}, \quad i=1 \ldots i_{a}$

$W_{i}=f_{a}\left(U_{i}\right), \quad i=i_{a}+1 \ldots N$

Combining (14a-b) and (15a-b), one gets:

$X_{i}=h\left(W_{1}\right), \quad i=1 \ldots i_{a}$

$X_{i}=h \circ f_{a}\left(U_{i}\right), \quad i=i_{a}+1 \ldots N$

These define the (asymptotic) system behaviour of the system (8a-b) when considering an ascendant series of constant-input experiments. This ascendant stage thus realized is graphically represented by plotting the points with coordinates $\left(U_{i}, X_{i}\right)(i=1 \ldots N)$. Doing so, one gets the ascendant path $\mathcal{P}_{a}$ of Fig. 2.

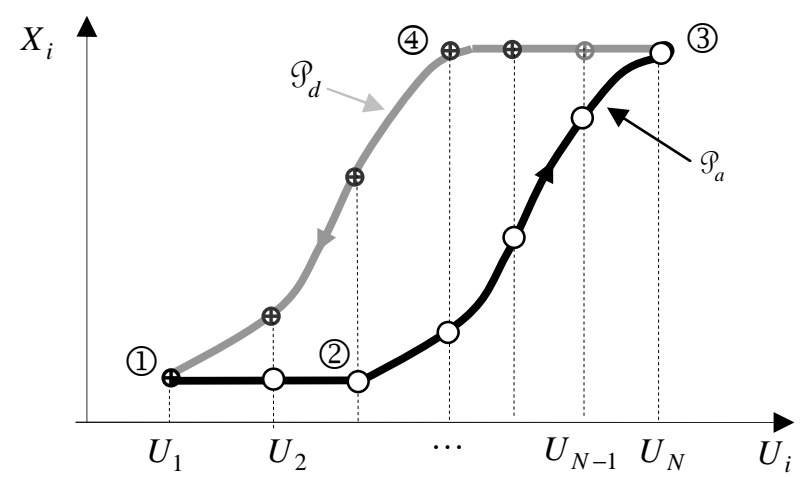

Fig. 2. Limit cycle described by the set of points $\left(U_{i}, X_{i}\right)$, obtained when the system (8a-b) is excited by constant inputs. The points represented by circles characterize the ascendant stage experiments and, all together, form the ascendant path $\mathscr{P}_{a}$. The points represented by crosses characterize the descendant stage experiments and, all together, form the descendant path $\mathscr{P}_{d}$.
Descendant experimental stage. After the above ascendant stage, one goes ahead realizing the descendant experiment series. Specifically, the system is successively excited with constant inputs with decreasing amplitudes $U_{N-1}<U_{N-2}<\ldots<U_{1}$. Following a similar argument as previously, it can be proved that the system asymptotic behaviour is described by the following equations, with $i=N-1 \ldots 1$ :

$u(t)=U_{i} \Rightarrow v(t) \approx w(t) \approx W_{i}, \quad x(t) \approx X_{i}=h\left(W_{i}\right)$

And, there is an integer $1 \leq i_{d} \leq N-1$ such that:

$X_{i}=h\left(W_{N}\right), \quad i=N-1 \ldots i_{d}$

$X_{i}=h \circ f_{d}\left(U_{i}\right), \quad i=i_{d}-1 \ldots 1$

The descendant stage is graphically represented by the points with coordinates $\left(U_{i}, X_{i}\right)(i=N-1 \ldots 1)$, defined by (18a-b).

These points form the descendant path $\mathscr{P}_{d}$ of Fig. 2 .

3.2 Determination of the functions $f_{a}, f_{d}, h$

Estimation of the coefficients of $h \circ f_{a}$ and $h \circ f_{d}$

The union $\mathscr{P}_{a} \cup \mathcal{P}_{d}$ of the two paths form a limit cycle of the compound operator $B\left(h \circ f_{a}, h \circ f_{d}\right)$. Then, it follows from (16b) that, the coefficients of the compound polynomial hof can be obtained using the following subset of $\mathscr{P}_{a}$ :

$\left\{\left(U_{i}, X_{i}\right) \in \mathscr{P}_{a} ; i=i_{a} \ldots N\right\}$

That is, except for those located on the horizontal segment (1)-(2), all points of $\mathscr{P}_{a}$ are used in the determination of $h o f_{a}$. Such determination is simply performed using the least squares algorithm, based on (7a) and (7c) which entail the following structure of $h o f_{a}$ :

$h \circ f_{a}(x)=\alpha_{n m} x^{n m}+\ldots+\alpha_{1} x+\alpha_{0}$

Similarly, it follows from (9b) that, the coefficients of the compound polynomial $h o f_{d}$ can be obtained using the following subset of $\mathscr{P}_{d}$ :

$\left\{\left(U_{i}, X_{i}\right) \in \mathscr{P}_{d} ; i=1 \ldots i_{d}-1\right\}$

Accordingly, all points of $\mathscr{P}_{d}$ are used in the determination of $h o f_{d}$, except for those located on the horizontal segment (3)(4). Again, the least squares algorithm is used, based on (17b) and $(17 \mathrm{c})$ which entail the following structure of $h o f_{d}$ :

$h \circ f_{d}(x)=\delta_{n m} x^{n m}+\ldots+\delta_{1} x+\delta_{0}$

Obviously, the exact determination of the $\alpha_{i}$ 's using the set of points (19) and the determination of the $\delta_{i}$ 's using (21) is possible provided that the number of points is sufficient, compared of the number of unknown coefficients. Specifically, it is required that:

$N-i_{a} \geq n m$ and $i_{d}-1 \geq n m+1$

Given a number $N>n m$, a practical rule to meet (23) consists in letting:

$U_{1}<<U_{2}$ and $U_{N-1}<<U_{N}$

Doing so, it is possible to meet the following properties:

$i_{a}=1$ and $i_{d}=N-1$

In view of Fig. 2, the first equality in (25) means that there is no (useless) point of $\mathscr{P}_{a}$ within the segment (1)-(2) or, in other 
words, the extremities of that segment are the points $\left(U_{1}, X_{1}\right)$ and $\left(U_{2}, X_{2}\right)$. Similarly, the second equality in (25) means that there is no (useless) point of $\mathscr{P}_{d}$ within the segment (3)-(4) i.e. the extremities of that segment are the points $\left(U_{N}, X_{N}\right)$ and $\left(U_{N-1}, X_{N-1}\right)$.

\section{Estimation of the coefficients of $\left(f_{a}, f_{d}, h\right)$}

Under conditions (23), the coefficients of the compound polynomials $h o f_{a}$ and $h o f_{d}$ can be uniquely determined, by the least squares algorithm, using the available set of points (25) and (21).

Now, applying the Kozen-Landau decomposition algorithm of Table 1 to the monic polynomial $\frac{1}{\alpha_{n+m}} h \circ f_{a}$ one gets a pair of two monic polynomials $\left(\bar{f}_{a}, \bar{h}\right)$ of the form,

$\bar{f}_{a}(x)=x^{n}+\bar{a}_{n-1} x+\ldots+\bar{a}_{1} x+\bar{a}_{0}$

$\bar{h}(x)=x^{m}+\bar{b}_{m-1} x^{m-1} \ldots+\bar{b}_{1} x+\bar{b}_{0}$

such that $\frac{1}{\alpha_{n m}} h \circ f_{a}=\bar{h} \circ \bar{f}_{a}$ or, equivalently:

$h \circ f_{a}=\alpha_{n m} \bar{h} \circ \bar{f}_{a}$

From Proposition 1 (Part 2), one gets that the pair of polynomials $\left(\bar{f}_{a}, \alpha_{n m} \bar{h}\right)$ is not unique but the set of solutions can be expressed in terms of the above pair. Specifically, for any pair on the form:

$\overline{\bar{f}}_{a}(x)=\bar{\gamma} \bar{f}_{a}(x)+\bar{\gamma} \bar{\beta}$ and $\overline{\bar{h}}(x)=\alpha_{n+m} \bar{h}\left(\frac{x}{\bar{\gamma}}-\bar{\beta}\right)$,

one has,

$\overline{\bar{h}} \circ \overline{\bar{f}}_{a}=h o f_{a}$,

whatever the scalars $\bar{\beta}$ and $\bar{\gamma} \neq 0$. But, in view of (12b) the most judicious choice consists in letting:

$\bar{\gamma}=1$ and $\bar{\beta}=-\bar{a}_{0}$

Doing so, one enforces $\overline{\bar{f}}_{a}(x)$ to be, just as $f_{a}(x)$, a monic polynomial with null lower degree term coefficient (see (12b)). Since one constructively has $\overline{\bar{h}} \circ \overline{\bar{f}}_{a}=h \circ f_{a}$, it follows from Proposition 1 (Part 2) that $\overline{\bar{f}}_{a}=f_{a}$ and $\overline{\bar{h}}=h$. Based on this result, it turns out that an exact estimation $\left(\hat{f}_{a}, \hat{h}\right)$ of $\left(f_{a}, h\right)$ is obtained as follows:

$\hat{f}(x)=\bar{f}(x)-\bar{a}_{0}$ and $\hat{h}(x)=\alpha_{n m} \bar{h}\left(x+\bar{a}_{0}\right)$

where $\left(\bar{f}_{a}, \bar{h}\right)$ are as in (26a-b).

Similarly, applying the Kozen-Landau decomposition algorithm to the monic polynomial $\frac{1}{\delta_{n m}} h o f_{d}$ one gets a pair of two monic polynomials $\left(\tilde{f}_{d}, \tilde{h}\right)$ of the form,

$\tilde{f}_{d}(x)=x^{n}+\tilde{d}_{n-1} x+\ldots+\tilde{d}_{1} x+\tilde{d}_{0}$

$\tilde{h}(x)=x^{m}+\tilde{b}_{m-1} x^{m-1} \ldots+\tilde{b}_{1} x+\tilde{b}_{0}$ such that $\frac{1}{\delta_{n m}} h \circ f_{d}=\tilde{h} \circ \tilde{f}_{a}$ or, equivalently:

$h \circ f_{d}=\delta_{n m} \tilde{h} \circ \tilde{f}_{d}$

From Proposition 1 (Part 2), it follows that the pair of polynomials $\left(\tilde{f}_{d}, \delta_{n m} \tilde{h}\right)$ is not unique as any pair of the form,

$\tilde{\tilde{f}}_{d}(x)=\tilde{\gamma} \tilde{f}_{d}(x)+\tilde{\gamma} \tilde{\beta}$ and $\tilde{\tilde{h}}(x)=\delta_{n m} \tilde{h}\left(\frac{x}{\tilde{\gamma}}-\tilde{\beta}\right)$,

is also a solution, i.e.

$\tilde{\tilde{h}} \circ \tilde{\tilde{f}}_{d}=h \circ f_{d}$

where $\tilde{\beta}$ and $\tilde{\gamma} \neq 0$ are any scalars. The key point is to get benefit of the freedom offered by these scalars to make $\tilde{\tilde{h}}(x)$ in (32a) equal to $\hat{h}(x)$ which is now entirely available. The second equations in (29) and (23), together with the expressions (30b) and (26b), show that $\tilde{\widetilde{h}}(x)=\hat{h}(x)$ if, and only if:

$$
\begin{aligned}
& \delta_{n m}\left(\left(\frac{x}{\tilde{\gamma}}-\tilde{\beta}\right)^{m}+\tilde{b}_{m-1}\left(\frac{x}{\tilde{\gamma}}-\tilde{\beta}\right)^{m-1} \ldots+\tilde{b}_{1}\left(\frac{x}{\tilde{\gamma}}-\tilde{\beta}\right)+\tilde{b}_{0}\right) \\
& =\alpha_{n m}\left(\left(x+\bar{a}_{0}\right)^{m}+\bar{b}_{m-1}\left(x+\bar{a}_{0}\right)^{m-1} \ldots+\bar{b}_{1}\left(x+\bar{a}_{0}\right)+\bar{b}_{0}\right)
\end{aligned}
$$

This leads to $m$ equations but there are only two unknown parameters i.e. $\tilde{\beta}$ and $\tilde{\gamma}$. The latter are readily obtained by letting the coefficients of the two highest degree terms on both sides be equal. Doing so, one gets:

$\frac{\delta_{n m}}{\tilde{\gamma}^{m}}=\alpha_{n m}$

$\frac{\delta_{n m}}{\tilde{\gamma}^{m-1}}\left(-m \tilde{\beta}+\tilde{b}_{m-1}\right)=\alpha_{n m}\left(m \bar{a}_{0}+\bar{b}_{m-1}\right)$

From (33a) one gets the value of $\tilde{\gamma}$ using the fact that $\operatorname{sgn}\left(\delta_{n m}\right)=\operatorname{sgn}\left(\alpha_{n m}\right)$. The latter holds because $\operatorname{sgn}\left(a_{n}\right)=\operatorname{sgn}\left(d_{n}\right)$ due to (4). Then, the parameter $\tilde{\beta}$ is simply obtained from equation (34b).

Tuning $\tilde{\beta}$ and $\tilde{\gamma}$ according to (34a-b), ensures that $\tilde{\tilde{h}}(x)=\hat{h}(x)=h(x)$. Then, using Proposition 1 (Part 2), one gets from (32b) that $\tilde{\tilde{f}}_{d}(x)=f_{d}(x)$. Based on this result, it turns out that an exact estimation $\hat{f}_{d}$ of $f_{d}$ is obtained as follows:

$$
\hat{f}_{d}(x)=\tilde{\gamma} \tilde{f}_{d}(x)+\tilde{\gamma} \tilde{\beta}
$$

where $\tilde{f}_{d}$ is as in (34b) and $(\tilde{\gamma}, \tilde{\beta})$ is the solution of (34a-b).

\section{LINEAR SUBSYSTEM IDENTIFICATION}

At this point, the input and output nonlinearities, $F[$.$] are$ $h($.$) , are known and the aim is to determine the transfer$ function $G(r)(r=s$ or $z)$ of the linear subsystem. In this respect, recall that, by Assumption A2, one has $\operatorname{sgn}\left(a_{n}\right)=\operatorname{sgn}\left(d_{n}\right) \quad$ which implies that the polynomial 
borders, $f_{a}($.$) and f_{d}($.$) , are asymptotically monotonic with$ similar monotony. Accordingly, there exist scalars $0 \leq u_{f}<\infty$ such that the borders are similarly monotonic on $\left[u_{f}, \infty\right)$ and similarly monotonic (with possibly opposite monotony sense) on $\left(-\infty,-u_{f}\right]$. At this stage $f_{a}($.$) and$ $f_{d}$ (.) are known and so one of such scalars can be explicitly determined. Then, Proposition 2 (Part 2) ensures that $F[$ [.] is invertible from the right on the interval $\left[u_{f}, \infty\right)$ and its rightinverse, denoted $F^{-1}[$.$] is equal either to B I\left(f_{a}^{-1}, f_{d}^{-1}\right)$ or $B I\left(f_{d}^{-1}, f_{a}^{-1}\right)$, depending on the monotony sense of $f_{a}($. and $f_{d}\left(\right.$.) on $\left[u_{f}, \infty\right)$. Then, one key idea is to neutralize the effect of $F[$.] by placing its inverse as pre-compensator (Fig. $3)$ and excite the augmented system with input signals $z(t)$ that only take values in the domain where $F \circ F^{-1}=I$. Specifically, one has:

$$
z(t) \in f_{a}^{-1}\left(\left[u_{f}, \infty\right)\right) \cap f_{d}^{-1}\left(\left[u_{f}, \infty\right)\right), \forall t \Rightarrow v(t)=z(t), \forall t
$$

Similarly, the output nonlinearity $h($.$) is asymptotically$ monotonic, due to its polynomial nature. Let $0 \leq w_{h}<\infty$ be any scalar such that $h($.$) is invertible on \left[w_{h}, \infty\right)$ and on $\left(-\infty, w_{h}\right]$ and let its inverse on $h\left(\left[w_{h}, \infty\right)\right) \cap h\left(\left(-\infty, w_{h}\right]\right)$ be denoted $h^{-1}($.$) . Then, our second key idea is to choose the$ input signal $z(t)$ so that the observed output signal $x(t)$ is enforced to stay all the time in either $h\left(\left[w_{h}, \infty\right)\right)$ or $h\left(\left(-\infty, w_{h}\right]\right)$. This can be achieved by letting $u_{f}$ in (35) be sufficiently large, whatever the nature of $z(t)$. In such an operation mode, the effect of the output nonlinearity can be cancelled by placing the element $h^{-1}$ (.) as post-compensator (Fig. 3). Doing so, the augmented system, including both the pre- and post-compensator, boils down to a linear system with transfer function $G(r)$. The fact that the input signal $z(t)$ is of arbitrary nature entails the possibility of choosing it to be persistently exciting making the problem of identifying $G(r)$ a trivial issue.

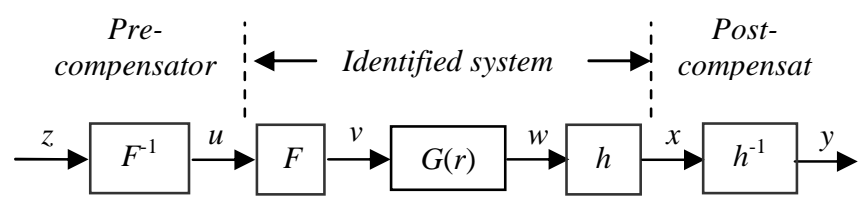

Fig. 3. The system to be identified augmented with pre- and post-compensator

\section{CONCLUSION}

The problem of identifying Hammerstein-Wiener systems is addressed in presence of backlash input nonlinearities and memoryless output nonlinearities. All system nonlinearities are parametric and not-necessarily invertible. The linear subsystem may be parametric or not, continuous- or discretetime. A two-stage identification approach is developed to deal with this problem. Accordingly, the nonlinear parts are determined first using the Kozen-Landau polynomial decomposition. This stage necessitates simple constant-input experiments. The linear subsystem identification is coped with by using pre- and post-compensators designed so that the augmented system reduces to the linear part of the initial Hammerstein-Wiener system.

\section{REFERENCES}

Abrahamsson, R., S.M. Kay, and P. Stoica (2007). Estimation of the parameters of a bilinear model with applications to submarine detection and system identification. Digital Signal Processing, 17(4), 756-773.

Crama, P. and J. Schoukens (2004). Hammerstein-Wiener system estimator initialization. Automatica, 40(9), 1543-1550.

Bai E.W. (2010). An optimal two-stage identification algorithm for Hammerstein-Wiener Nonlinear Systems. In Giri and Bai. Blockoriented Nonlinear System Identification. Springer, U.K., 27-34.

Bai E. W. (2002). A Blind Approach to the Hammerstein-Wiener Model Identification. Automatica, 38 (6), 967-979.

Giri F. and E.W. Bai (2010). Block-Oriented Nonlinear System Identification. Springer, U.K.

Giri F., Y. Rochdi, A. Brouri, and F.Z. Chaoui (2010). Frequency identification of Wiener systems with backlash nonlinearity. In IFAC Symposium on Nonlinear Control Systems, 368-373. Bologna, Italy.

Kozen D. and S. Landau (1989). Polynomial decomposition algorithms. $J$. Symbolic Computation, 7, 445-456.

Ni B., H. Garnier, and M. Gilson (2012). A refined instrumental variable method for Hammerstein-Wiener continuous-time model identification. In Proc. 16th IFAC Symp. Syst. Identification, Brussels, Belgium, pp. 1061-1066.

Palanthandalam-Madapusi, H.J., A.J. Ridley, and D.S. Bernstein (2005). Identification and prediction of ionospheric dynamics using a Hammerstein-Wiener model with radial basis functions. In Proc. of the American Control Conference, 5052-5057.

Schoukens, M., E.W. Bai, and Y. Rolain (2012). Identification of Hammerstein-Wiener Systems. In Proc. 16th IFAC Symp. Syst. Identification, 274-279. Brussels, Belgium.

Tao G., P. Kokotovic. Adaptive Control of Systems with Actuator and Sensor Nonlinearities. John Wiley And Sons Ltd, US, 1996.

Taringou, F., O. Hammi, B. Srinivasan, R. Malhame, and F.M. Ghannouchi (2010). Behaviour modelling of wideband RF transmitters using Hammerstein-Wiener models. IET Circuits Devices \& Systems, 4(4), 282-290.

Vörös, J. (2004). An iterative method for Hammerstein-Wiener systems parameter identification. Journal of Electrical Engineering, 55(11-12), 328-331.

Wang, D. and F. Ding (2008). Extended stochastic gradient identification algorithms for Hammerstein-Wiener ARMAX systems. Computers \& Mathematics with Applications, 56(12), 3157-3164. 